\title{
Optimizing ISOCAM data processing using spatial redundancy ${ }^{\star}$
}

\author{
M.-A. Miville-Deschênes ${ }^{1,2}$, F. Boulanger ${ }^{1}$, A. Abergel $^{1}$, and J.-P. Bernard ${ }^{1}$ \\ 1 Institut d'Astrophysique Spatiale, Université Paris-Sud, Bât. 121, 91405 Orsay, France \\ 2 Département de Physique, Observatoire Astronomique du Mont Mégantic, Université Laval, Sainte-Foy, Québec, G1K 7P4, \\ Canada
}

Received April 16, 1999; accepted August 11, 2000

\begin{abstract}
Several instrumental effects of the Long Wavelength channel of ISOCAM, the camera on board the Infrared Space Observatory, degrade the processed images. We present new data-processing techniques that correct these defects, taking advantage of the fact that a position in the sky has been observed by several pixels at different times. We use this redundant information (1) to correct the long-term variation of the detector response, (2) to correct memory effects after glitches and point sources, and (3) to refine the deglitching process. As an example we have applied our processing to the gamma-ray burst observation GRB 970402. Our new data-processing techniques allow the detection of faint extended emission with contrast smaller than $1 \%$ of the zodiacal background. The data reduction corrects instrumental effects to the point where the noise in the final map is dominated by the readout and the photon noises. All raster ISOCAM observations can benefit from the data processing described here. This includes mapping of solar system extended objects (comet dust trails), nearby clouds and star forming regions, images from diffuse emission in the Galactic plane and external galaxies. These techniques could also be applied to other raster type observations (e.g. ISOPHOT).
\end{abstract}

Key words: techniques: image processing — infrared: general - instrumentation: detectors

\section{Introduction}

The Infrared Space Observatory (ISO) of the European Space Agency ceased observations on April 81998 ,

Send offprint requests to: M.-A. Miville-Deschênes

* Based on observations with ISO, an ESA project with instruments funded by ESA Member States (especially the PI countries: France, Germany, The Netherlands and the UK) and with the participation of ISAS and NASA.

Correspondence to: e-mail: mamd@ias.fr
10 months after its expected end. This mission was a great success (Kessler 1999) and many of the resulting scientific discoveries are yet to come. Many of the scientific analyses of the data are still held up by data reduction problems. In particular for the camera on board ISO (ISOCAM - Cesarsky 1996), instrumental effects which are not well understood limit its sensitivity.

Much effort has been made to model the response of the ISOCAM array based on theoretical understanding of infrared detectors (Abergel 2000; Coulais \& Abergel 2000 and references therein). Nevertheless, no overall model of the ISOCAM response to flux steps and glitches is available. ISOCAM data reduction thus relies on an empirical understanding of the detector. Recently, sophisticated data reduction techniques have been developed to take into account some aspects of ISOCAM response (Starck et al. 1999; Desert et al. 1999; Aussel et al. 1999; Altieri et al. 1999). These methods are close to being optimal for the detection of point sources. Nevertheless, in many observations, instrumental effects still prevent the study of faint extended emission.

The development of the data reduction method presented in this paper was motivated by the analysis of ISOCAM observations of diffuse and translucent interstellar clouds. Such clouds, when illuminated by the solar neighborhood radiation field, have mid-infrared brightness with very low contrast with respect to the zodiacal light (at most a few percent). To reveal these low signal-to-noise ratio clouds, variations in the detector response have to be corrected to an accuracy better than a fraction of $1 \%$. At the present time, available data-processing techniques do not achieve such high sensitivity for extended emission. In this paper, we present a data processing method that makes it possible.

The algorithms presented here apply to observations made in raster mode with the Long Wavelength channel of ISOCAM (LW). It makes use of the fact that a given position in the sky has been observed several times by different camera pixels. Tests have been performed on 
extended emission observations presenting low and high contrasts. We have checked that our methods can be applied to most raster mode observations, and as a consequence concerns a significant fraction of ISOCAM observations (extra-galactic, galactic and even solar system observations).

To illustrate the data processing we use two different observations of the same field, obtained sequentially in exactly the same configuration. The amplitude of instrumental effects are not the same in both observations, giving us constraints on the validity of our method. In Sect. 2 we present the observations used to illustrate the data reduction chain. In Sect. 3 standard data reduction techniques are briefly presented. The main problems of the standard reduction are described in Sect. 4 and the new data processing approach to address these problems is detailed in Sect. 5. The performances of the overall method are discussed in Sect. 6.

\section{Observation}

The main ISOCAM technical characteristics are presented in (Cesarsky 1996). The LW channel of ISOCAM operates between 4 and $18 \mu \mathrm{m}$. A lens wheel allows the selection of the field of view per pixel (1.5, 3, 6 and 12 arcsecs), and a filter wheel the selection of the spectral band pass (10 broad band filters and two Continuously Variable Filters). The detector is a $500 \mu \mathrm{m}$ thick crystal made of gallium doped silicon photo-conductor hybridized by Indium bumps. The $32 \times 32$ square pixels are defined with a pitch of $100 \mu \mathrm{m}$.

A given observation (characterized by a given configuration (lens, filter, integration time)) is presented as a collection of $32 \times 32$ images (called readouts) gathered together in a data cube. Three different observational modes were available with ISOCAM (Siebenmorgen et al. 1997): 1) single pointing, raster mode, 2) beam switching and 3) spectrophotometry. The observations presented in this paper were obtained in raster mode where many readouts are put together to build a mosaic of the observed object (called the sky image). The scanning strategy is made in such a way that each readout taken on the sky has some overlap with its neighbors (see Fig. 1).

To illustrate the data reduction procedures, we use two ISOCAM observations of the gamma-ray burst GRB 970402 first observed by BeppoSAX (Feroci et al. 1997) on April 2.93 UT 1997. The coordinates (J2000) of the burst were $\alpha=14^{\mathrm{h}} 50^{\mathrm{m}} 16^{\mathrm{s}}, \delta=-69^{\circ} 19^{\prime} \cdot 9$ with an error circle of radius $3^{\prime}$. Target-of-opportunity ISO observations (ISOCAM and ISOPHOT) were requested by Castro-Tirado et al. (1998) to detect a transient infrared emission of this burst. Unfortunately, the observations did not show such emission. Nevertheless, these particular observations are of great interest for us since the same field was observed twice, subsequently within the same

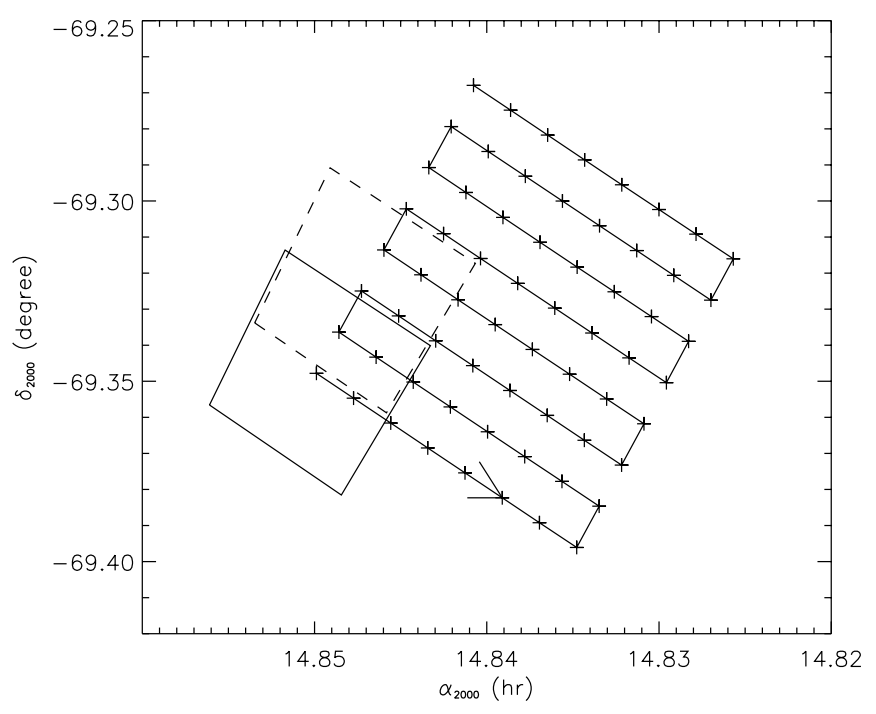

Fig. 1. Raster observing strategy. The crosses indicate the positions on the sky of the camera center. The first (solid line) and the sixteenth (dash line) positions of the camera are shown as square. The arrow shows the scanning direction

orbit, in exactly the same configuration, to look for a decrease in the GRB emission. The observational strategy of the observations is sketched in Fig. 1 and described in Table 1. Comparing the result of our processing on both observations (GRB1 and GRB2 in the following) allows us to validate the method.

\section{Building the standard low level sky map}

The standard ISOCAM reduction method consists of the following steps:

Deglitching. On any observation, numerous energetic particle impacts leave traces on one or several pixels of the detector. Most of these impacts do not affect the detector response on long time scale and are seen as instantaneous flux step (fast glitches). In Fig. 2, a typical flux history of one pixel as a function of time is shown where many fast glitches are apparent. Various techniques are used to suppress these emission spikes (Starck 1999).

Dark image subtraction. The response of the LW detector of ISOCAM is not zero when the detector does not receive any photons coming from the sky. This dark image must be subtracted from the data. Temporal variation of the dark image has been observed (Starck et al. 1999) and modeled by Biviano et al. (1998) using a polynomial function of time.

Short term transient. It has been known since pre-launch measurements (Perault et al. 1994) that the ISOCAM detector is affected by a transient effect, i.e. its response to a given flux step is not instantaneous. Recently a new model of the response, based on the physic of Si:Ga arrays (Fouks \& Schubert 1995), has been 
Table 1. Journal of the two ISOCAM observations of the gamma-ray burst GRB 970402

\begin{tabular}{lll}
\hline & GRB1 & GRB2 \\
\hline \hline Date & April 5 1997 & April 5 1997 \\
ISO Revolution & 506 & 506 \\
Time since activation & $2.12(\mathrm{hrs})$ & $3.42(\mathrm{hrs})$ \\
Filter & LW10 & LW10 \\
Wavelength & $8.5-15.0 \mu \mathrm{m}$ & $8.5-15.0 \mu \mathrm{m}$ \\
Right Ascension J2000 & $14 \mathrm{~h} 50 \mathrm{~m} 18.2 \mathrm{~s}$ & $14 \mathrm{~h} 50 \mathrm{~m} 18.1 \mathrm{~s}$ \\
Declination J2000 & $-69^{\circ} 19^{\prime} 57.1^{\prime \prime}$ & $-69^{\circ} 19^{\prime} 59.3^{\prime \prime}$ \\
Total number of readouts & 907 & 907 \\
Number of raster steps & $8 \times 8$ & $8 \times 8$ \\
Readouts per positions & 12 & 12 \\
Step size (pixels) & 8 & 8 \\
Exposure time (seconds) & 5.04 & 5.04 \\
Pixel size & $6^{\prime \prime}$ & $6^{\prime \prime}$ \\
Comment & Strong LTT & Good quality \\
\hline
\end{tabular}
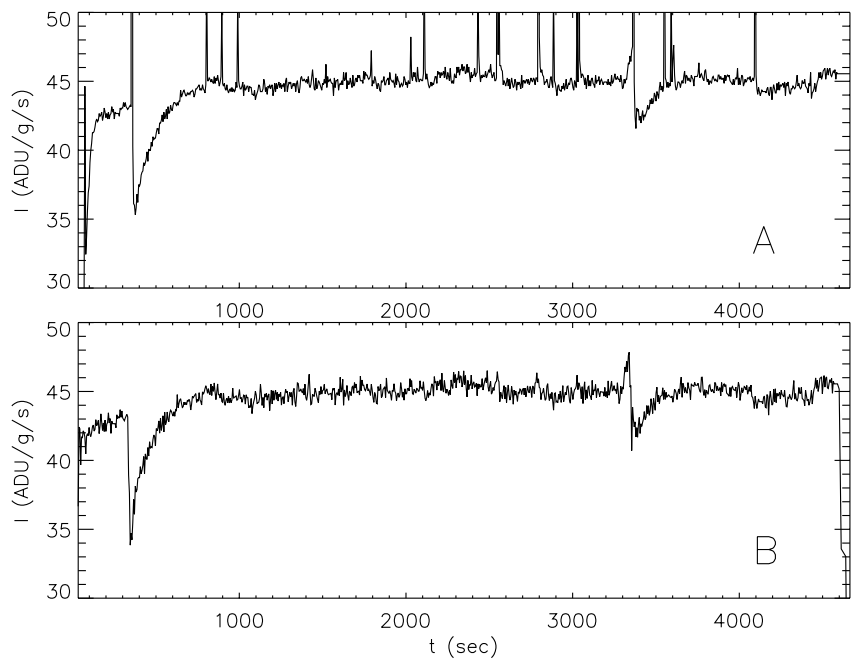

Fig. 2. Temporal evolution of a typical pixel. A) Raw data many energetic electrons have hit the pixel causing instantaneous flux steps known as fast glitches. A response accident is apparent near $t=350 \mathrm{~s}$ caused by an ion impact (slow glitch). B) Fast glitch corrected data - short time flux steps have been identified as glitches and removed from the data cube

developed by Coulais \& Abergel (2000). The uncertainty in the corrected flux with this new model is $\sim 1 \%$ but raises to about $10 \%$ for point sources, likely due to charge coupling effects between neighboring pixels. This is not a strong problem here since we are mostly interested in diffuse emission.

Flat Field. Generally, each pixel of a detector does not have exactly the same response to a given flux. This spatial variation of the detector response (the flat field in the following) must be taken into account. The most straightforward way to compute an image of the flat field is to average all readouts of a data cube, and normalize the mean to 1 (Starck et al. 1999). This technique supposes that all camera pixels have seen the same average flux along the observation. It is a reasonable approximation in raster mode where the camera is moving on a region of the sky much larger than the field of view of the camera, which does not contain any systematic gradient.

Building the sky image. After dividing the data cube by the flat field, the final standard data reduction step is to project each readout onto the sky to build the sky image. The LW channel of ISOCAM is affected by a field of view distortion problem that must be taken into account in this operation (Starck et al. 1999), especially to properly add emission from point sources and small scale structures.

The sky image of the GRB1 observation obtained with the standard data processing described in this section is shown in Fig. 8A. It is affected by many instrumental problems that limit the full sensitivity of the instrument. In the next section we describe the open problems for the imaging of faint extended emission.

\section{Open problems}

Long term transient. Figure 3 presents the temporal evolution of the median flux observed in the $11 \times 11$ central square of the detector for the GRB1 (a) and GRB2 (b) observations. One striking feature of Fig. 3 is the drift in the first observation, not seen in the second. This drift in ISOCAM response is observed quite frequently and is called in the following the Long Term Transient (LTT). When observed, the amplitude of the LTT is about 5\% of the total sky emission, as in Fig. 3. One also sees that the first sky image (Fig. 8A) is completely dominated by the long term drift. This is true for any observation affected by a LTT of faint extended emission with contrasts smaller than a few percent of the zodiacal light.

The LTT was identified during the pre-launch measurements. Many calibration observations presented a slow and continuous increase of the response after a positive flux step. This rise gradually attenuates on a variable time 

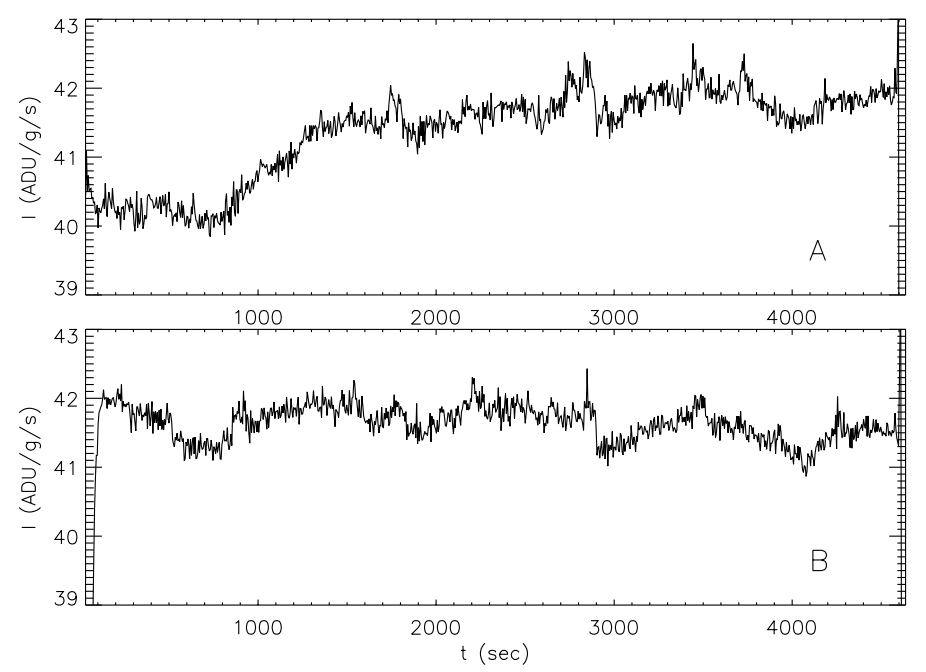

Fig. 3. Temporal evolution after short term transient correction of the median flux observed in the $11 \times 11$ central part of the camera, for the GRB1 A) and GRB2 B) observations

scale which can be up to several hours. Unlike the short term transient, no analytical or physical description has been developed so far to correct for this specific effect which seems to be a general behavior of this type of detector. Vinokurov \& Fouks (1991) addressed the problem of long term drift in their study of nonlinear response of photo-conductors. Unfortunately, the analytical approximation they propose does not seem to reproduce accurately ISOCAM's LTT.

Slow glitches. Another important problem is related to glitches which modify the response during a significant time. This is illustrated in Fig. 2 where one notices a significant change in the detector response near $t=350 \mathrm{~s}$, just after a glitch impact. The glitch impact is removed by the standard deglitching algorithm but the detector response is significantly disrupted for more than $500 \mathrm{~s}$. In this example the signal is depressed after the glitch, but it can also be enhanced. These glitches with a memory effect (called in the following "slow glitches"), which affect the response of one or more pixels for some time after the impact, are believed to be due to heavy ions. They are responsible for most of the periodic patterns seen in Fig. 8A. No model has been developed to correct this instrumental effect. Désert et al. (1999) describe the various types of slow glitches known and a method to correct the response accident. However, it is not suitable for our problem; this method was indeed optimized for point source extraction where diffuse emission is not intended to be restored.

Ghosts. There are also several artificial point sources ("ghosts" in the following) due to uncorrected memory effects. After seeing a bright point source, the response of a given pixel is significantly affected for some time. We have seen in the previous section that significant memory effects remain after strong point sources even after the short term transient correction. Therefore, as the satellite moves on the sky from one sky position to the other, pixels that have observed bright point sources are affected by a memory effect, and the pattern of each point source appears repetitively in the sky image until the response of the pixels returns to a normal value.

We conclude that the actual data processing does not adequately take into account (1) the long term drift, (2) slow glitches and (3) short term transients on point sources. The long term drift precludes the study of largescale emission fainter than $10 \%$ of the zodiacal light background. All instrumental effects limit the brightness sensitivity for small scale structures. Solving these problems is crucial in order to take advantage of the unique sensitivity and angular resolution of ISOCAM. This is particularly true for small-scale structures since generally the contrast of interstellar medium emission decreases from large to small scales (Gautier 1992).

\section{New approach for LW-ISOCAM data processing}

\subsection{General description}

\subsubsection{General inversion method}

To address the problems described in Sect. 4 we have developed a method which uses the fact that a position on the sky has been observed by several ISOCAM pixels at different times (see Fig. 1). The redundant information is used to separate the contributions of the sky emission and of instrumental effects to the observed signal. This approach has already been used for the IRAS mission (e.g. Okumura 1991; Wheelock et al. 1997) and may be generalized to every raster type observations, whatever the wavelength of observation. Formally the processing of astronomical data with spatial redundancy could be treated as an inversion problem. We can consider that the data observed $O$ is the result of the convolution of the real sky $S$ with the instrumental function $I$ plus some additive noise $N$ :

$O=I \otimes S+N$.

When the observation has been conducted with spatial redundancy, it may be possible to address the data processing problem globally by finding $I, S$ and $N$ that will reproduce $O$ and minimize the difference between measurements obtained at the same sky position. Such an approach supposes that the main instrumental effects that affect $I$ and $N$ are known enough to constrain the inversion method. Presently this is not the case for ISOCAM.

\subsubsection{Sequential approach}

ISOCAM's response variations are complex and presently we are not able to model it with a reasonable number 


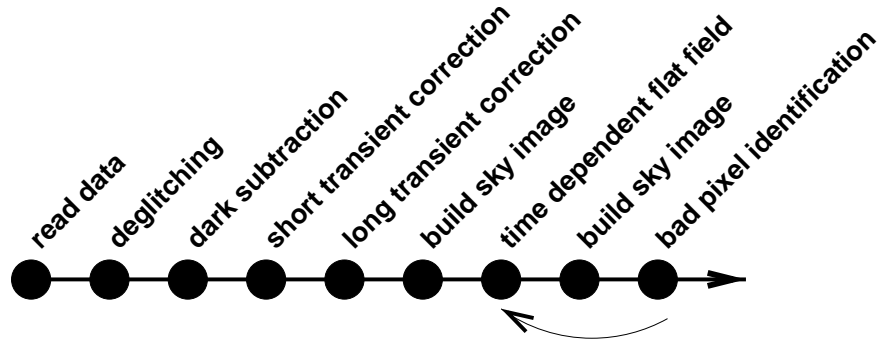

Fig. 4. Data reduction chain illustrating the data processing steps from left to right. The time dependent flat field and bad pixel identification operations can be done iteratively

of parameters which would allow us to address the data processing problem by a global inversion. We have thus adopted a sequential approach where the instrumental effects are treated one at a time. Nevertheless, even if we cannot use such a global method, the idea of minimizing the differences between pixels that have seen the same sky position is the mainstay of our approach. In particular, the ISOCAM long term drift problem is addressed by a least square minimization technique based on the fact that, if the detector is slowly reaching stabilization, the measured flux is also approaching the real observed flux. Here we suppose that every pixels of the array is affected by the same long term drift. The implication of this assumption will be discussed in Sect. 6 .

Once the LTT is removed, the other response variations (slow glitches, ghosts and residual transient effects) are corrected by comparing the data cube to the sky image. This is done in two steps. First we compute a variable flat field that takes into account pixel-to-pixel response variations. Second, pixels that have a flux that departs significantly from the sky image are flagged (these are called bad pixels in the following). This last operation removes slow glitches and ghosts. These two operations (variable flat field and bad pixel identification) can be done several times to improve the sky image (see Fig. 4).

\subsection{Long term transient}

\subsubsection{Gain or Offset effect?}

First of all it is important to determine how the long term transient, the flat field $F$ and the observed flux $I_{\text {obs }}$ are related to the incident flux $I_{\text {sky }}$. We have considered the three following possibilities for the LTT:

1. Gain effect: $I_{\mathrm{obs}}=G(t) F I_{\text {sky }}$;

2. Offset effect affected by flat: $I_{\mathrm{obs}}=F\left(I_{\mathrm{sky}}+\Delta(t)\right)$;

3. Pure offset effect: $I_{\mathrm{obs}}=F I_{\text {sky }}+\Delta(t)$.

The two GRB observations are of great help in identifying the nature of the LTT. As the two observations were done in exactly the same way, it is possible to subtract directly the two data cubes. In Fig. 5A we show the flux history of

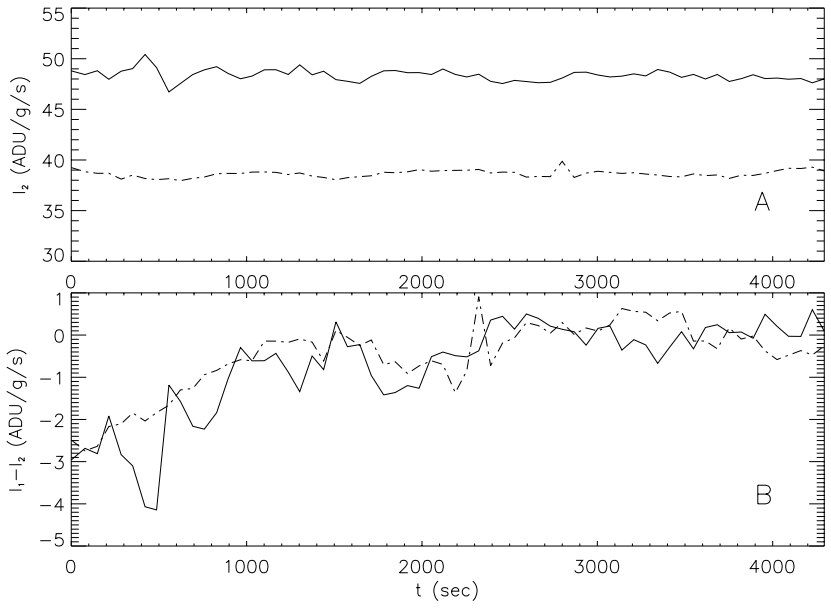

Fig. 5. A) Flux history of two pixels of the GRB2 observation. This observation is not affected by the LTT. The flux difference between the two pixels is due to an intrinsic response difference (flat field). B) Difference between the two GRB observations for the two pixels considered in A). Even if the two pixels have very different responses, the LTT amplitude is the same

two pixels of the GRB2 observation. As one can see there is no long term drift detectable in this observation. The flux difference between the two selected pixels is due to a $\sim 25 \%$ flat field difference. The difference between observation 1 and 2 for these two pixels is shown in Fig. 5B. Both pixels have a very similar drift; the $25 \%$ difference between the two pixels flux does not appear in Fig. 5B. We then conclude that the LTT does not depend on the signal $F I_{\text {sky }}$. In this context, the only valid description of the LTT is the pure offset effect: $I_{\text {obs }}=F I_{\text {sky }}+\Delta(t)$. In the following we consider the LTT as a single offset over all the detector; in other words we suppose that all pixels are affected by the same offset.

\subsubsection{Formalism}

For a given pixel at a position $(x, y)$ on the detector array and at a given time $t$, the observed flux $I_{\mathrm{obs}}(x, y, t)$ is related to the temporally varying flat field $F(x, y, t)$, the incident flux $I_{\text {sky }}(x, y, t)$ and the long term drift $\Delta(t)$ by the following equation:

$I_{\text {obs }}(x, y, t)=F(x, y, t) I_{\text {sky }}(x, y, t)+\Delta(t)$.

Here we suppose that $\Delta(t)$ is not pixel dependent.

The offset function $\Delta(t)$ is found using Eq. (2) and the spatial redundancy inherent to raster mode observations. We determine $\Delta(t)$ by solving a set of linear equations obtained by comparing flat field corrected intensities of the same sky positions but obtained at different times. The flat field $F(x, y, t)$ is computed using the adaptive flat field method (see Appendix A.2). The flat field corrected intensities can be written:

$\frac{I_{\mathrm{obs}}(x, y, t)}{F(x, y, t)}=I_{\mathrm{sky}}(x, y, t)+\frac{\Delta(t)}{F(x, y, t)}$. 
In raster mode the camera does not always point at the same position on the sky. To compare two pixels that have seen the same position on the sky, we must put all readouts in a common spatial reference frame. Practically, we project $I_{\mathrm{obs}}(x, y, t)$ and $F(x, y, t)$ on the plane of the sky (right ascension $(\alpha)$ - declination $(\delta)$ reference frame). In this reference frame and following Eq. (3), the difference between two pixels that have seen the same sky $I_{\text {sky }}(\alpha, \delta)$ at different time $\left(t_{i}\right.$ and $\left.t_{j}\right)$ is:

$\frac{I_{\mathrm{obs}}\left(\alpha, \delta, t_{i}\right)}{F\left(\alpha, \delta, t_{i}\right)}-\frac{I_{\mathrm{obs}}\left(\alpha, \delta, t_{j}\right)}{F\left(\alpha, \delta, t_{j}\right)}=\frac{\Delta\left(t_{i}\right)}{F\left(\alpha, \delta, t_{i}\right)}-\frac{\Delta\left(t_{j}\right)}{F\left(\alpha, \delta, t_{j}\right)} \cdot(4)$ To ensure that we compare fluxes obtained at the same sky position, the field of view distortion must be accurately taken into account in the projection operation.

The function of interest $\Delta(t)$ is estimated using a leastsquare minimization technique with the following minimization criterion:

$$
\begin{aligned}
\chi^{2}= & \sum_{\alpha, \delta, t_{i}, t_{j}}\left[\frac{I_{\mathrm{obs}}\left(\alpha, \delta, t_{i}\right)}{F\left(\alpha, \delta, t_{i}\right)}-\frac{I_{\mathrm{obs}}\left(\alpha, \delta, t_{j}\right)}{F\left(\alpha, \delta, t_{j}\right)}\right. \\
& \left.-\frac{\Delta\left(t_{i}\right)}{F\left(\alpha, \delta, t_{i}\right)}+\frac{\Delta\left(t_{j}\right)}{F\left(\alpha, \delta, t_{j}\right)}\right]^{2} .
\end{aligned}
$$

Here the sum is over all the possible pixel pairs that have seen the same region of the sky.

The function $\Delta(t)$ which minimizes the value of $\chi^{2}$ is found by solving the system determined by:

$$
\frac{\partial \chi^{2}}{\partial \Delta\left(t_{i}\right)}=0 \text {. }
$$

Equation (6) represents a standard set of linear equations which can be written in a matrix form: $A \Delta(t)=B$, with:

$$
\begin{aligned}
& A(i, j)_{i \neq j}=\sum_{\alpha, \delta} \frac{1}{F\left(\alpha, \delta, t_{i}\right) F\left(\alpha, \delta, t_{j}\right)} \\
& A(i, j)_{i=j}=\sum_{\alpha, \delta, t_{j \cap i}} \frac{-1}{F\left(\alpha, \delta, t_{i}\right) F\left(\alpha, \delta, t_{j}\right)} \\
& B(i)=\sum_{\alpha, \delta, t_{j \cap i}} \frac{1}{F\left(\alpha, \delta, t_{i}\right)}\left[\frac{I_{\mathrm{obs}}\left(\alpha, \delta, t_{j}\right)}{F\left(\alpha, \delta, t_{j}\right)}-\frac{I_{\mathrm{obs}}\left(\alpha, \delta, t_{i}\right)}{F\left(\alpha, \delta, t_{i}\right)}\right] .
\end{aligned}
$$

The $A(i, j)$ and $B(i)$ terms are computed with all pixels that overlap. For each pair $(i, j)$, the sums are always computed on the positions $(\alpha, \delta)$ where $I_{\mathrm{obs}}\left(\alpha, \delta, t_{i}\right)$ and $I_{\mathrm{obs}}\left(\alpha, \delta, t_{j}\right)$ are both defined.

Finally $\Delta(t)$ is found from: $\Delta(t)=A^{-1} B$. As the second derivative of $\chi^{2}$ is always positive, the solution found for $\Delta(t)$ necessarily minimizes the $\chi^{2}$ criterion. We add an offset to all values of $\Delta(t)$ to force the correction to be zero at the end of the observation: $\Delta\left(t_{\text {end }}\right)=0$. This is justified by the fact that the long term transient tends to stabilize at the end of an observation. However, there are several observations not stabilized at the end, so that the absolute brightness may be systematically shifted (generally below a few \%). For the observations of low contrasted clouds on top of a flat large scale emission (at least the zodiacal emission), this point is not critical as we always remove the large scale emission at the end of our processing.

\subsubsection{Practical implementation}

Other than detector noise, two additional sources of uncertainties affect the comparison of raster images and thus the LTT correction through the minimization algorithm described in the previous section: slow glitches and flat field variations along the observation. The signal measured on point sources is not identical in the individual readouts, essentially due to the undersampling of the the point spread function for the $6^{\prime \prime}$ pixels. Bright sources are thus an additional source of error but they can easily be discarded. The two other noise sources make the practical implementation of the formalism a non-straightforward procedure.

An extensive use of the LTT inversion method presented here on real data has demonstrated the extreme importance of an accurate flat field. As the LTT correction is based on the comparison of the brightness measured by different parts of the array, its result depends on the accuracy of the flat field. In particular, when $F(\alpha, \delta, t)$ is not well estimated, oscillations in phase with the rastering of the observations may appear in the correction found. To overcome this difficulty we compute an approximate LTT correction (as oppose to the exact correction which is the solution of Eq. (6)). The study of low contrast ISOCAM data affected by long term drifts has shown that, in some cases, the LTT can be approximated by the sum of two exponential functions, one upward and one downward:

$\Delta(t) \approx P \times \exp \left(-Q \times t^{R}\right)-S \times \exp \left(-T \times t^{U}\right)$

where $P, Q, R, S, T$ and $U$ are strictly positive. By approximating the LTT in this way, we greatly simplify the problem as $\Delta(t)$ now depends only on six parameters. As for the exact solution, the approximated solution is found by minimizing the criterion of Eq. (5), using an IDL curve fitting program (MPFIT). The obvious advantage of this method is that the approximated LTT correction is smooth, getting rid of the oscillations found in some exact solutions.

On the other hand, we have observed cases where this approximation of the LTT does not hold. Sometimes, the LTT shows oscillations that can dominate the emission in low contrasted fields (e.g. cirrus clouds - see MivilleDeschênes et al. 2000). In these cases, an accurate estimation of the flat field and the use of the exact solution for the LTT are mandatory.

\subsubsection{LTT correction for the GRB1 observation}

Figure 6 presents the LTT corrections found for the GRB1 observation. A adaptive single flat field (see Appendix A.2) was used and point sources were discarded to compute the exact and approximated corrections. The approximated LTT correction is smoother than the exact one, which oscillates with $\mathrm{a} \sim 0.2 \mathrm{ADU} / \mathrm{g} / \mathrm{s}$ amplitude and a period of $\sim 1800 \mathrm{~s}$. In this case we have applied the approximated 


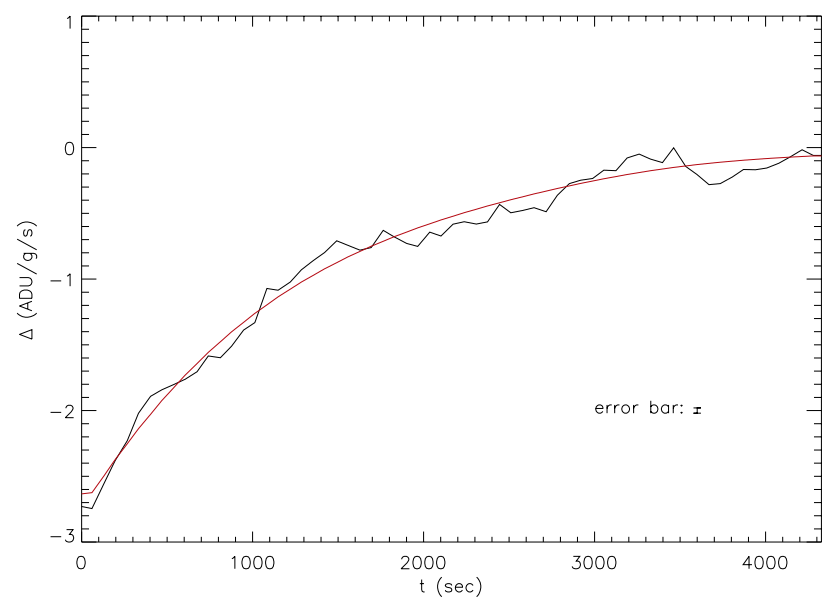

Fig. 6. Exact and approximated LTT correction for the GRB1 observation. Estimating the error on the LTT correction is difficult as it is very sensitive to the accuracy of the flat field used. The error bar represented here is the statistical error (0.08 ADU/g/s - see Appendix B). A more realistic error determination would be related to the accuracy of the flat field For the GRB observations, a $1 \%$ uncertainty on the flat field corresponds to a $0.4 \mathrm{ADU} / \mathrm{g} / \mathrm{s}$ error on the LTT correction

LTT correction. The sky image computed after that correction has been done is presented in Fig. 8B. One sees that it is mandatory to apply the LTT correction since its amplitude $(3 \mathrm{ADU} / \mathrm{g} / \mathrm{s})$ is nearly three times the amplitude of the emission of interest $(1.1 \mathrm{ADU} / \mathrm{g} / \mathrm{s})$. At this stage we have used a single flat field to compute the sky image. It is necessary to use a variable flat field to correct the artefacts (e.g. periodic patterns) seen in Fig. 8B.

\subsection{Variable Flat Field}

After the LTT has been corrected, we then take into account the pixel-to-pixel temporal variations of the detector response. These response variations are observed at various timescales. At short timescales they are due to bad short term transient correction, particularly on point sources. On longer time scales they are mainly caused by slow glitches (see Sect. 4). The use of a single flat field (see Sect. 3) does not take into account these temporal variations (that represent $\sim 1-3 \%$ of the average flat field) preventing the benefit of the optimal ISOCAM sensitivity. To go further in the data processing, we try to correct these pixel-to-pixel response variations with a time-dependent flat-field (called "variable flat field" in the following).

For LTT corrected data, the observed flux $I_{\text {obs }}$ at position $(x, y)$ on the array and at time $t$ is

$I_{\mathrm{obs}}(x, y, t)=F(x, y, t) I_{\mathrm{sky}}(x, y, t)$,

where $F(x, y, t)$ is the instantaneous response of pixel $(x, y)$ at time $t$. Thus flat field and sky structures are mixed together in $I_{\mathrm{obs}}(x, y, t)$. In the following we show

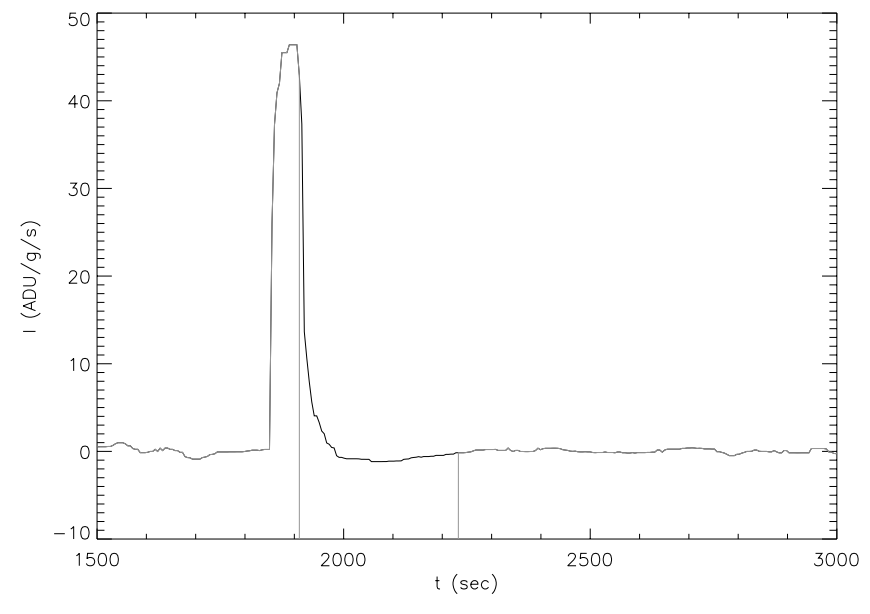

Fig. 7. Ghost identification. Flux history of a pixel which has observed a point source. The flux values after the point source are affected by a memory effect and are identified as a ghost (flagged out)

how the flat field variations can be extracted from the data by estimating $I_{\text {sky }}(x, y, t)$ and by taking advantage of the spatial redundancy.

In raster observation mode, many pixels of the data cube have seen the same position $(\alpha, \delta)$ on the sky. By averaging all these pixels we reduce the noise due to instrumental effects on the computation of the sky image $I_{\text {sky }}(\alpha, \delta)$. The estimate of $I_{\text {sky }}(x, y, t)$ is made by an inverse projection of $I_{\text {sky }}(\alpha, \delta)$ on each readout of the data cube. Then $F(x, y, t)$ is computed by averaging $I_{\mathrm{obs}}(x, y, t) / I_{\mathrm{sky}}(x, y, t)$ with a sliding window on the time axis (see Appendix A.1). Here are the guidelines of this method:

1. Construct a sky image;

2. Smooth (median smoothing) the sky image with a $10 \times 10$ window;

3. Compute an ideal cube $I_{\text {sky }}(x, y, t)$ by projecting the smoothed sky image on each readout of the data cube;

4. Smooth (median smoothing) $I_{\mathrm{obs}}(x, y, t) / I_{\mathrm{sky}}(x, y, t)$ on the time axis. The size of the smoothing window should be of the order of the time spent on 5 different sky positions.

The sky image of the GRB1 observation, obtained with the variable flat field, is shown in Fig. $8 \mathrm{C}$. The size of the filtering window on the time axis is 100 (corresponding to $\sim 500 \mathrm{~s}$ ). As seen in Fig. $8 \mathrm{C}$, the variable flat field removes almost all periodic patterns due to high-frequency variations of the detector response. Compared to other methods we have tested (see Appendices A.1 and A.2), this variable flat field gives by far the best results.

\subsection{Bad pixel identification}

The deglitching process used at the beginning of the reduction chain (see Sect. 3) removes extremely deviant pixels 


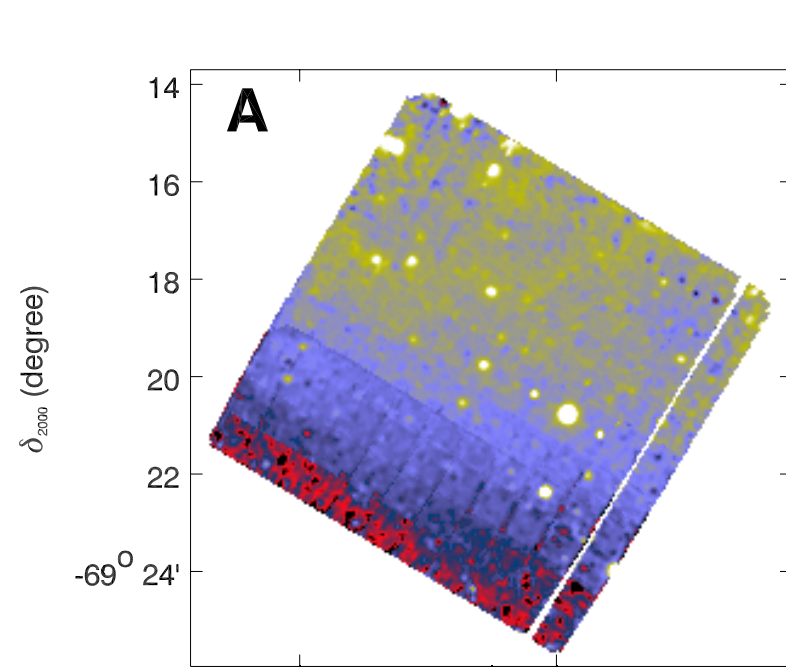

$\mathrm{ADU} / \mathbf{g} / \mathbf{s}$

$\mathrm{ADU} / \mathbf{g} / \mathbf{s}$
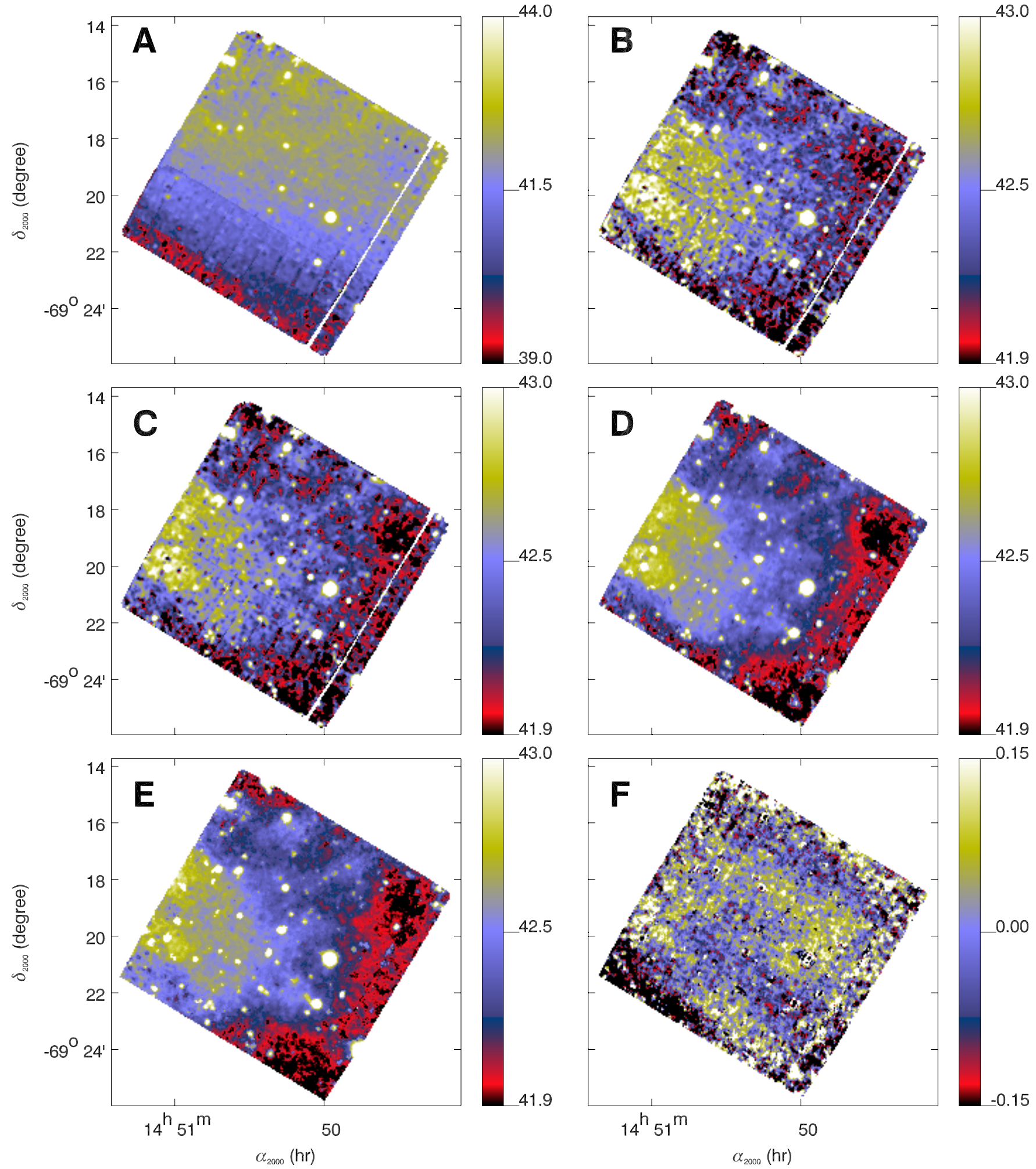

Fig. 8. LW10 images of a the first GRB 970402 observation after deglitching, dark subtraction, transient correction A), long term transient correction B), variable flat field C) and bad pixel removal D). Image E) is the final map of the second GRB 970402 observation and image $\mathbf{F}$ ) is the difference between $\mathbf{D}$ ) and $\mathbf{E}$ ). For these observations, $1 \mathrm{ADU} / \mathrm{g} / \mathrm{s}$ corresponds to $0.242 \mathrm{mJy} /$ pix or $0.286 \mathrm{MJy} / \mathrm{sr}$ 


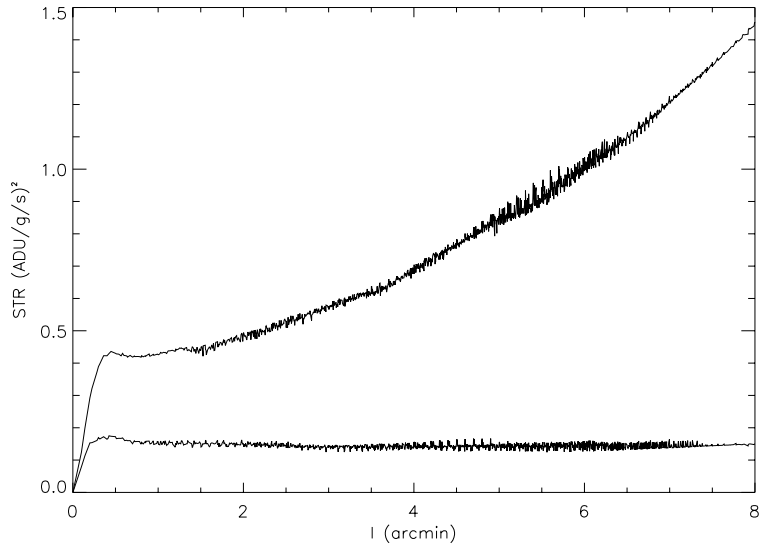

Fig. 9. Structure function of the difference between the two GRB final maps (bottom line) and of the difference between the first map of GRB1 and the final map of GRB2 (top line)

that have been hit by cosmic rays. To go further in the noise minimization process, we again take advantage of the spatial redundancy.

\subsubsection{Ghost removal}

Memory effects often appear after a strong flux step (e.g. after a point source) due to improper short term transient correction; this is what we call ghosts. To identify pixels affected by such effects, we look at the flux history of every pixel for a memory effect after a flux step (see Fig. 7). Again, we use the redundancy information to improve the identification of ghosts. Here is how we proceed:

1. Smooth (median smoothing) the sky image with a $3 \times$ 3 window;

2. Compute an ideal cube by projecting the smoothed sky image on each readout of the data cube;

3. Compute the residual cube $=\mid$ data cube - ideal cube $\mid$;

4. Identify strong flux steps as residual flux above $10 \times$ the noise level;

5. On the time history of each pixel, identify a ghost as a residual flux above or under the noise level after $a$ strong flux step;

6. Flag ghosts in the original data cube.

\subsubsection{Noise reducer}

We can go further in the reduction of the noise level by working sky position by sky position instead of working on the time history of every pixel. The idea is to look at pixels in the data cube that have seen the same sky position and discard deviant flux values.

First the sky image is smoothed (median smoothing) with a $10 \times 10$ window. Then, for a given sky position $(\alpha, \delta)$, we compare the $N$ pixels in the data cube that have seen that position with the flux of the smoothed sky image. For most of the sky position, the $N$ pixels are distributed around the smoothed sky estimate. On the other hand, at point source positions, the $N$ pixels generally will be above the smoothed sky estimate. Furthermore, it is also possible to find sky positions where most of the $N$ pixels have fluxes under the smoothed sky level. Here is how we deal with each case:

1. Most of the $N$ pixels are around the smoothed sky level $(S M L)$;

The new sky flux is the average of $(S M L-3 \times$ noise $<$ $I<S M L+3 \times$ noise);

2. Most of the $N$ pixels are above the smoothed sky level; The new sky flux is the average of $I>S M L$;

3. Most of the $N$ pixels are under the smoothed sky level; The new sky flux is the average of $(S M L-3 \times$ noise $<$ $I<S M L)$.

This method allows us to globally reduce the noise level and to keep a good photometry of point sources. This is the final data processing step. The sky images obtained after bad pixel removal of the first and second GRB observations are presented in Figs. 8D and 8E.

\section{Assessment of the method}

\subsection{Comparison of the two GRB observations}

From the comparison of the final maps of the two GRB observations (Figs. 8D and 8E) we can estimate the reliability of our processing. At first glance we see that the structure of the diffuse emission is very similar in both maps; the LTT correction applied seems to restore properly the large scale structure. Furthermore, almost all point like structures are present in both maps, giving confidence in our bad pixel identification.

The difference of the two final sky images is shown in Fig. 8F. It is dominated by small-scale structure noise but large-scale structures are also apparent. These are probably due to error in the LTT correction. Extra noise is seen at point source positions. This was expected as memory effects are not fully corrected on point sources and as we are undersampling the point spread function (PSF). One also notices that the noise level is higher at the edges of the difference map, due to less redundancy in these regions. The standard deviation of the difference map (Fig. 8F) in the central part of the field is $0.06 \mathrm{ADU} / \mathrm{g} / \mathrm{s}$. Therefore, the noise on each GRB final map can be estimated at $0.06 / \sqrt{2}=0.04 \mathrm{ADU} / \mathrm{g} / \mathrm{s}$.

It is clear from the difference map (Fig. 8F) and from the impact of the LTT on the sky image that noise is present at all scales. The processing presented in this paper affects the signal at various scales. To characterize the noise as a function of angular scale we use the structure function of second order:

$B^{2}(l) \equiv \frac{\sum\left[I_{\mathrm{sky}}(r)-I_{\mathrm{sky}}(r+l)\right]^{2}}{N(l)}$, 


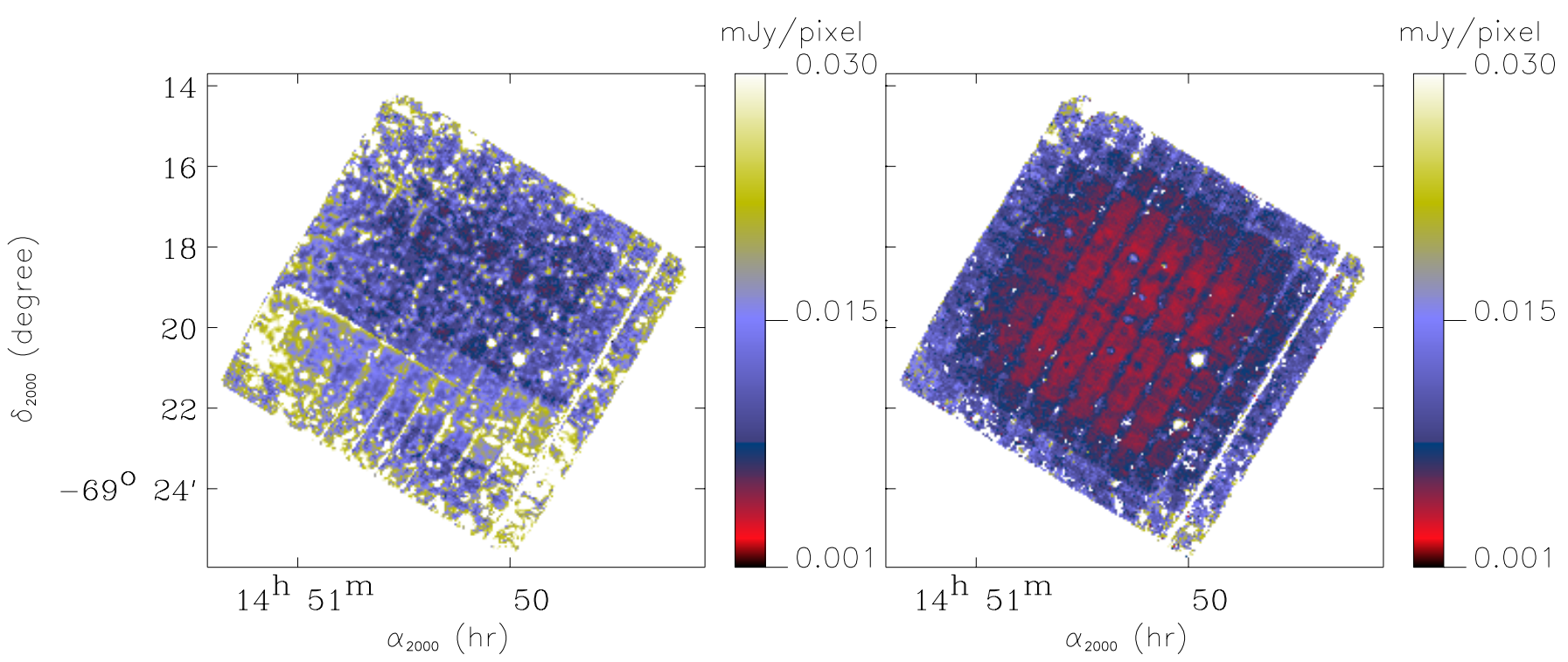

Fig. 10. Noise maps before (left) and after (right) the processing. For this observation $1 \mathrm{ADU} / \mathrm{g} / \mathrm{s}$ corresponds to $0.242 \mathrm{mJy} / \mathrm{pix}$

where the sum is over all the $N(l)$ pairs of points separated by a distance $l$. To estimate the noise at each scale in the first map, the one affected by all instrumental effects, we compute the structure function on the difference between the first map of the GRB1 observation and the final one of the GRB2 observation. This difference map, where the sky is removed, is dominated by the noise of the GRB1 observation. The structure function of this difference map rises strongly from small to large scale (see Fig. 9), mainly due to the presence of the LTT. To estimate the noise at the end of the processing, we have computed the structure function on the difference between the final maps of the two GRB observations (see Fig. 8F). This time the structure function is very flat (see Fig. 9). The noise level is reduced by a factor of ten at 8 arcmin scale and a factor of two at the resolution limit. This indicates that the noise level has been lowered at all scales and that it is now uniform at all scales.

\subsection{Study of the noise sources}

The goal of this section is to show that the high spatial frequency noise of our maps is close to the optimal value obtained with stabilized ground calibration data with no glitches. The data are affected by many sources of noise. First, there are the classical quantum photon noise and the detector readout noise which have been extensively studied in the pre-launch calibration phase (Perault et al. 1994). A conservative value of the readout noise is given in the ISOCAM cookbook: $1.5 \mathrm{ADU} / \mathrm{g}$. Secondly, memory effects (short term transient, long term transient and slow glitches) and fast glitches with small amplitudes substantially increase the noise level. These non-Gaussian events may prevent to reach the optimal sensitivity.
The noise level is measured on the flux history of pixels for which fast glitches have been removed. We have selected pixels not affected by slow glitches. We quantify the noise using the standard deviation of the high frequency component of the pixel flux history. We see in Table 2 that the noise level of the two GRB observations is in total agreement with the readout and photon noise estimated from calibration data (for a $41.5 \mathrm{ADU} / \mathrm{g} / \mathrm{s}$ flux).

The flux $I_{\text {sky }}$ at a given position in the final sky image is the average of $N$ independent flux measurements. The error $\delta$ on $I_{\text {sky }}$ can be estimated by

$\delta=\frac{\sigma}{\sqrt{N}}$

where $\sigma$ is the standard deviation of the $N$ flux measurements used to compute $I_{\text {sky }}$. We have computed the error map at each step of the processing. In Fig. 10 we show the error $\delta$ of each sky position for the sky image obtained before the LTT correction (Fig. 10A) and for the final sky image (Fig. 10B) of the GRB1 observation. In the Fig. 10A one sees an enhancement of the error in the southern part of the image, due to the presence of the LTT. Glitches and periodic flat defects appear as noise peaks in this error map. The structure of the final error map (Fig. 10) is dominated by the redundancy effect: the noise is higher on the edges of the map as less flux measurements were obtained in these regions.

For each sky image of Fig. 8, Table 2 lists the median error $\delta$, the median redundancy $N$ and the median standard deviation of the $N$ flux measurements averaged at each sky position. The first thing to notice is that the noise level decreases gradually through the processing. In the final sky image, the median $\sigma$ is only $5 \%$ above the noise calibration measurement for the GRB1 observation. The noise in the GRB2 observation is exactly the one obtained with stabilized ground calibration data with no glitches. 
Table 2. The noise level in the sky image at each step of the processing. In comparison, the noise level measured on the flux history of a single pixel of observation GRB1 and the noise level predicted by ground calibrations (for a mean flux of $I=41.5 \mathrm{ADU} / \mathrm{g} / \mathrm{s}$ ) are both $1.15 \mathrm{ADU}$ (which corresponds to $7.26 \mu \mathrm{Jy})$

\begin{tabular}{l||l|l|l|l}
\hline & Sky Image & $\delta^{a}$ & $N^{b}$ & $\sigma^{c}$ \\
\hline GRB1 & Before LTT correction & 0.062 & 76 & 2.72 \\
& After LTT correction & 0.053 & 76 & 2.32 \\
& Variable Flat Field & 0.046 & 76 & 2.01 \\
& Final map & 0.031 & 58 & 1.21 \\
\hline GRB2 & Final map & 0.030 & 60 & 1.15 \\
\cline { 2 - 5 }$a$ & Median error value of each sky image (in ADU $/ \mathrm{g} / \mathrm{s}$ ). \\
$b$ & Median redundancy of each sky image (in number of \\
readouts). \\
${ }^{c} \sigma$ (in ADU) $=$ Error $\times \sqrt{\text { Redundancy }} \times$ Integration time.
\end{tabular}

The dispersion of the difference between the two final maps (divided by $\sqrt{2}$ ) is $0.04 \mathrm{ADU} / \mathrm{g} / \mathrm{s}$ (see Sect. 6.1) which is $35 \%$ above the noise level computed on each final map (0.03 ADU/g/s - see Table 2). This 35\% difference is partly due to the increased noise on point sources and to the imperfection of the LTT correction. Nevertheless, considering the amplitude of the instrumental effects (3 ADU $/ \mathrm{g} / \mathrm{s}$ for the LTT) we think that the comparison between the two GRB observations is a strong validation of the whole processing.

Finally we conclude from the numbers of Table 2 that the noise level in our final maps are dominated by the readout and photon noise. The other instrumental effects are corrected in the data reduction. Such ISOCAM sensitivity has been obtained in recent point source extraction studies (Desert et al. 1999; Aussel et al. 1999) where the low frequency diffuse emission is removed. It is the first time that such a sensitivity is reached for the emission structure on all scales.

\section{Conclusion}

The data processing techniques presented here are based on the fact that each position on the sky has been observed several times and by different pixels along the observation. Taking advantage of this redundant information inherent to raster mode observations, we are able to control some instrumental effects which degrade the signal, reduce the noise level and finally bring images obtained with the LW channel of ISOCAM to a quality level close to optimal. The principal of the method presented could be generalized to all observations with spatial redundancy.

The quality of the final images is limited by our ability to separate the various instrumental effects. We have adopted a sequential approach where each of the problems is treated one after the other, but a global minimization would be a better way to find the optimal so- lution. However, such an approach seems to be out of reach for ISOCAM since several instrumental problems (e.g. the LTT and response variation after glitches and point sources) are presently not understood.

All raster ISOCAM observations can benefit from the data processing described here. This includes mapping of solar system extended objects (e.g. comet dust trails), nearby clouds and star forming regions, images from diffuse emission in the Galactic plane and external galaxies. Several publications based on the interpretation of ISOCAM observations reduced by the present method are being prepared.

Acknowledgements. We thank the different teams at IAS, CEA-Saclay, ENS-Paris and ESA for their outstanding work and continuous support during all phases of the ISO project. The Fond FCAR du Québec and the National Science and Engineering Research Council of Canada provided funds to support this research project. MAMD warmly thanks Leo Metcalfe for very helpful discussions and Do Kester for his judicious comments.

\section{Appendix A: Flat fielding}

\section{A.1. Averaging on a sliding window}

One way to estimate a flat field for each readout of a data cube consists of computing a sliding window average of the data cube:

$F\left(x, y, t_{i}\right)=\frac{1}{N} \sum_{t_{j}=t_{i}-N / 2}^{t_{i}+N / 2} I_{\mathrm{obs}}\left(x, y, t_{j}\right)$,

where $N$ is the size of the sliding window.

The variable flat field is normalized to 1 over the $11 \times 11$ central part of $F(x, y, t)$ :

$F\left(x, y, t_{i}\right)=\frac{F\left(x, y, t_{i}\right)}{\left\langle F\left(10: 21,10: 21, t_{i}\right)\right\rangle}$.

Here we suppose that during the $N$ readouts the camera has observed many positions on the sky and each pixel of the detector has seen the same flux on average. This is a good approximation if the sky observed is rather smooth and does not contain too much small-scale structure. If the emission is fairly uniform on the detector, the number of images required to compute the flat field may be relatively small. The choice of $N$ depends on the number of readouts taken per sky position and on the contrast of small scale structures (in our case we use $N=100$ ). Extreme high and low flux pixels were rejected (top and bottom 15\%) in order to exclude glitches and point sources. The main limitation of this method is that sky structures may be still present in the variable flat field. 


\section{A.2. Adaptive flat field}

The adaptive single flat field method is based on the following assumption. We suppose that the variable flat field is, to a first order, a single flat field $F(x, y)$ computed on the whole data cube (see Sect. 3 ). The temporal variations of the flat field are treated as perturbations of the single flat, and we have:

$I_{\mathrm{obs}}(x, y, t)=[1+\delta(x, y, t)] F(x, y) I_{\text {sky }}(x, y, t)$,

where $\delta(x, y, t)$ is the perturbation term that take into account flat field deformations.

Flat deformations are mainly due to slow glitches and bad short term transient correction (especially on point sources). Therefore, for one given readout, they are considered as small scale defects, and $\delta(x, y, t)$ is dominated by high frequency structures in the $(x, y)$ space.

On a large-scale, the quantity

$\frac{I_{\mathrm{obs}}(x, y, t)}{F(x, y)}=I_{\mathrm{sky}}(1+\delta(x, y, t))$

is dominated by real large scale structures $\left(I_{\text {sky }}(x, y, t)\right)$ and not by flat defects $(\delta(x, y, t))$.

The low frequency sky emission $I_{\mathrm{SLF}}(x, y, t)$ is estimated by smoothing (median filtering) each readout of $I_{\mathrm{obs}}(x, y, t) / \mathrm{Flat}(x, y)$. The size of the smoothing window has to be smaller than the smallest sky structures and larger than the largest flat defects. In most cases, a compromise must be found (typically a $7 \times 7$ window). The flat field deformations then are estimated using:

$(1+\delta(x, y, t)) \simeq \frac{I_{\mathrm{obs}}(x, y, t)}{F(x, y) I_{\mathrm{SLF}}(x, y, t)}$.

We get rid of residual high frequency real sky structures by applying a temporal sliding average (see Appendix A.1) over the right hand side term of Eq. (A5).

The variable flat field is finally obtained with:

$\operatorname{Flat}(x, y, t)=[1+\delta(x, y, t)] F(x, y)$.

The averaging on a sliding window method (see Appendix A.1) follows low frequency temporal flat field deformations but, as a limited number of readouts are averaged together, sky structures are still present in the flat field. The adaptive single flat field is a better estimate since low frequencies of the incident flux cube are removed.

\section{Appendix B: LTT error determination}

The error on the LTT correction is estimated using the standard deviation constructed with all the pixel pairs used to compute $\Delta(t)$. The offset correction is based on Eq. (4). For a pixel pair $\left(\left(\alpha, \delta, t_{i}\right)\right.$ and $\left.\left(\alpha, \delta, t_{j}\right)\right)$ that have seen the same sky at different time, the offset at time $t_{i}$ is given by:

$$
\begin{aligned}
\Phi\left(\alpha, \delta, t_{i}, t_{j}\right)= & F\left(\alpha, \delta, t_{i}\right) \times \\
& {\left[\frac{I_{\mathrm{obs}}\left(\alpha, \delta, t_{i}\right)}{F\left(\alpha, \delta, t_{i}\right)}-\frac{I_{\mathrm{obs}}\left(\alpha, \delta, t_{j}\right)}{F\left(\alpha, \delta, t_{j}\right)}+\frac{\Delta\left(t_{j}\right)}{F\left(\alpha, \delta, t_{j}\right)}\right] . }
\end{aligned}
$$

We have evaluated the statistical error on the offset correction $\Delta\left(t_{i}\right)$ :

$\sigma\left(t_{i}\right)=\sqrt{\frac{1}{n(n-1)} \sum_{\alpha, \delta, t_{j \cap i}}\left(\Delta\left(t_{i}\right)-\Phi\left(\alpha, \delta, t_{i}, t_{j}\right)\right)^{2}}$

where $n$ is the number of pixel pairs.

\section{References}

Abergel A., Miville-Deschênes M.A., Désert F.X., et al., 2000, Exp. Astron. 10, 353

Altieri B., Metcalfe L., Kneib J.P., et al., 1999, A\&A 343, L65

Aussel H., Cesarsky C.J., Elbaz D., Starck J.L., 1999, A\&A 342,313

Biviano A., Sauvage M., Roman P., Boulade O., Gallais P.O.K., 1998, ESA report

Castro-Tirado A.J., Metcalfe L., Laureijs R., et al., 1998, A\&A 330,14

Cesarsky C.J., Abergel A., Agnese P., et al., 1996, A\&A 315, L32

Coulais A., Abergel A., 2000, A\&AS 141, 533

Désert F.X., Puget J.L., Clements D.L., 1999, A\&A 342, 363

Feroci M., Costa E., Piro L., et al., 1997, IAU Circ. 6610, 1

Fouks B.I., Schubert J., 1995, Proc. of SPIE 2553, 461

Gautier T.N.I., Boulanger F., Perault M., Puget J.L., 1992, AJ 103, 1313

Kessler M.F., 1999, in The Universe as Seen by ISO, Cox P., Kessler M.F (eds.), ESA-SP 427, p. 23

Miville-Deschênes M.A., Boulanger F., Abergel A., 2000, in ISO Beyond Point Sources: Studies of Extended Infrared Emission, R.J. Laureijs, K.L., Kessler M.F (eds.), ISO Data Centre, Villafranca del Castillo, Madrid, Spain, p. 111

Okumura K., 1991, Annales de Physique, supplement au Colloque No. 3, 16, 40

Pérault M., Désert F.X., Abergel A., et al., 1994, Opt. Eng. 33,762

Siebenmorgen R., Starck J.L., Cesarsky D.A., Guest S., Sauvage M., 1997, Isocam data users manual. Technical report, ISO Science Operations Center, Madrid

Starck J.L., Abergel A., Aussel H., et al., 1999, A\&AS 134, 135

Vinokurov L.A., Fouks B.I., 1991, Sov. Phys. Semicond. 25, 1207

Wheelock S., Gautier T.N., Chillemi J., et al., 1997, Issa explanatory supplement, Infrared Processing and Analysis Center 